\title{
Volumetric photoacoustic endoscopy of internal organs: a phantom and in situ study
}

Joon-Mo Yang, Konstantin Maslov, Ruimin Chen, HaoChung Yang, Qifa Zhou, et al.

Joon-Mo Yang, Konstantin Maslov, Ruimin Chen, Hao-Chung Yang, Qifa Zhou, K. Kirk Shung, Lihong V. Wang, "Volumetric photoacoustic endoscopy of internal organs: a phantom and in situ study," Proc. SPIE 7564, Photons Plus Ultrasound: Imaging and Sensing 2010, 75640D (23 February 2010); doi: 10.1117/12.842503

SPIE. Event: SPIE BiOS, 2010, San Francisco, California, United States 


\title{
Volumetric photoacoustic endoscopy of internal organs: a phantom and in situ study
}

\author{
Joon-Mo Yang ${ }^{1}$, Konstantin Maslov ${ }^{1}$, Ruimin Chen ${ }^{2}$, Hao-Chung Yang ${ }^{2}$, Qifa Zhou ${ }^{2}$, K. Kirk \\ Shung ${ }^{2}$, and Lihong V. Wang ${ }^{*}$ \\ ${ }^{1}$ Optical Imaging Laboratory, Department of Biomedical Engineering, Washington University in St. \\ Louis, One Brookings Drive, Campus Box 1097, St. Louis, Missouri, 63130, USA \\ ${ }^{2}$ Ultrasonic Transducer Resource Center, Department of Biomedical Engineering, University of \\ Southern California, 1042 Downey Way, University Park, DRB 130, Los Angeles, CA 90089, USA
}

\begin{abstract}
In this study, we further developed our photoacoustic endoscopic system to produce three-dimensional images of internal organs by performing pullback C-scans. Employing the side-scanning photoacoustic endoscopic probe discussed in the Optical Society of America's journal Optics Letters, we could acquire successive B-scan images by pulling back the probe with a motorized linear stage. We demonstrate the endoscopic system's volumetric imaging ability through imaging of a metal wire phantom and an in situ rat rectum.
\end{abstract}

Keywords: Photoacoustic endoscopy, volumetric imaging, tomography, internal organs, rectum, in situ imaging, optical biopsy.

\section{INTRODUCTION}

Tomographic images of internal organs have been extremely useful in clinical settings. These images can provide unprecedented depth information about target organs. Developing new endoscopic imaging modalities having such tomographic ability is one of important research directions because such technique could provide not only threedimensional structural (morphological) information but also functional information about suspicious tissue. Ultrasound endoscopy ${ }^{1-6}$ was the first embodiment with such tomographic imaging ability, and now this technique has matured enough that it is a common procedure. So far, the technique has shown to have broad application areas, with the ability to provide images of a wide variety of tissues, from the cardiovascular system ${ }^{1-4}$ to the gastrointestinal $\operatorname{tracts}^{5-6}$. Currently it is considered to be one of the strongest tomographic endoscopic imaging modalities in terms of image penetration depth. However, it has shown to have some weaknesses and defects as well. It demonstrates a serious ultrasound speckle artifact problem, and its mechanical property-based contrast mechanism prohibits it from detecting early cancer. For this reason, it is important that researchers develop new endoscopic modalities with high contrast and specificity, along with tomographic ability.

Various optical endoscopic imaging modalities have been reported in the biomedical optics field, and thus those techniques have sparked the formation of active research areas. Among these modalities, confocal endoscopy ${ }^{7-8}$ and endoscopic optical coherence tomography ${ }^{9-13}$ have been popular choices because their systems are relatively easy to implement in the form of endoscopic probe and they can provide high resolution imaging ability. Since these two techniques work at ballistic or quasi-ballistic regimes of photon migration with tight optical focusing, they can produce high resolution optical contrast images. However, although most tissue abnormalities, such as cancer, develop at the

*Corresponding author: lhwang@biomed.wustl.edu

Photons Plus Ultrasound: Imaging and Sensing 2010, edited by Alexander A. Oraevsky, Lihong V. Wang, Proc. of SPIE Vol. 7564, 75640D · @ 2010 SPIE · CCC code: 1605-7422/10/\$18 · doi: 10.1117/12.842503 
endothelial tissue level, the image penetration depths of these two techniques are not sufficient for encompassing the tissue region.

On the other hand, photoacoustic endoscopy ${ }^{14-16}$ based on photoacoustic tomography ${ }^{14,17-23}$ has great potential for in vivo medical application because it has deep imaging ability ${ }^{21}$ with strong optical absorption-based contrast. The image resolution can be scaled by appropriately choosing optical and ultrasonic parameters. Also, the technique is compatible with conventional ultrasound pulse-echo imaging because the two use the same type of ultrasonic transducer for ultrasound signal detection. In terms of contrast, photoacoustic endoscopy provides both structural and functional information at intrinsic contrast, and can derive plenty of exogenous contrast via various molecular probes ${ }^{22-23}$. Due to these strong capabilities, photoacoustic endoscopy is expected to make a significant impact in the medical imaging field.

In 2009, we introduced a photoacoustic endoscopic probe that was the first to integrate light illumination, ultrasonic detection, and a mechanical scanning mechanism into a small (4.2-mm dia.) tubular probe ${ }^{15-16}$. In this previous study, we demonstrated the probe's ability to produce B-scan (2D) images of biological tissue ex vivo or in situ. We also further developed the technique to perform C-scans, producing three-dimensional images of target organs. In this paper, we will describe the system implementation, provide experimental data, and include technical discussion of this technique.

\section{MATERIALS AND METHOD}

\subsection{Micromotor-based side-scanning photoacoustic endoscopic probe}

Figure 1(a) shows a schematic of our endoscopic probe's distal end, where a light-delivering optical fiber, photoacoustic signal sensor (ultrasonic transducer), and mechanical scanning unit are integrated ${ }^{15-16}$.

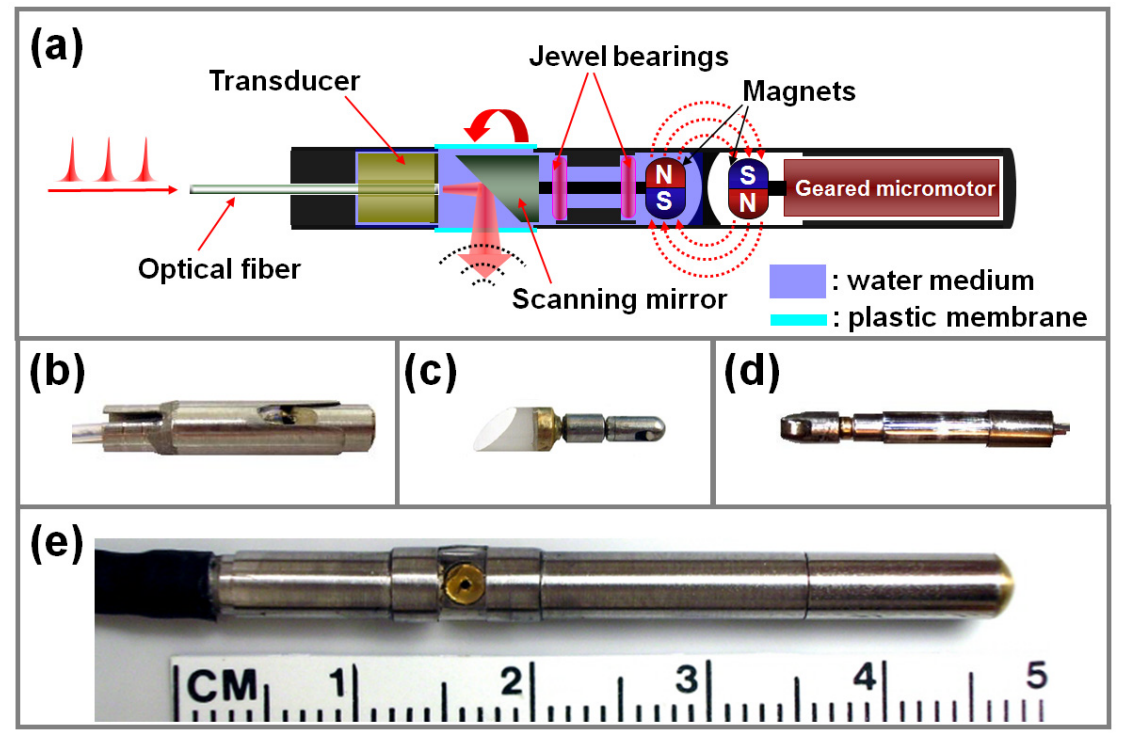

Figure 1: (a) Schematic of the photoacoustic endoscope's distal end (b) Optical fiber-ultrasonic transducer module (c) Scanning mirror module (d) Geared micromotor module (e) Photo of the photoacoustic endoscopic probe made in-house (distal end). The rigid distal end is $4.2 \mathrm{~mm}$ in diameter and $48 \mathrm{~mm}$ in length. Modules (b) through (d) are housed in the stainless steel tube shown in photo (e).

External laser pulses guided by an optical fiber are emitted though the central hole of the ultrasonic transducer, and then sent to the target tissue after reflection by an optical mirror (or scanning mirror). Finally the laser pulses generate photoacoustic waves by thermal expansion. Some of the photoacoustic waves propagate to the scanning mirror, reflect, and become detected by the ultrasonic transducer. Hence, the scanning mirror reflects both laser pulses and ultrasonic waves during its rotation, and performs radial scanning. Here, it is important to fill the probe's inner space with a liquid 
medium to provide an ultrasonic matching (propagation) medium for the photoacoustic waves. To perform mechanical scanning, we utilize a geared micromotor placed at the distal end of the probe. However, the micromotor's housing space is isolated from the liquid medium to provide in-air working condition; but the torque required for the mirror's rotation is transferred from the micromotor through a magnetic coupling.

The built-in mechanical source (micromotor) replaces the conventional flexible shaft-based mechanical scanning mechanism and enables radial scanning with static illumination optical fiber and ultrasonic sensor. By placing the optical fiber and ultrasonic transducer coaxially, we could achieve a uniform full B-scan.

Figure 1, images (b) through (d), presents the implementation of an optical fiber-ultrasonic transducer module, a scanning mirror module, and a micromotor module, respectively. Figure 1(e) shows the entire assembled endoscopic probe, which houses these modules. In the embodied probe, the transducer and scanning mirror's housing space are filled with deionized water and sealed with a plastic membrane (50 $\mu \mathrm{m}$ thick, LDPE) that is suitable for both light and ultrasonic wave transmission, and that forms an imaging window. The probe's maximal thickness is $4.2 \mathrm{~mm}$ at the plastic imaging window, and the total length of the rigid part is $48 \mathrm{~mm}$. The endoscope's flexible body is formed by three bundles of cables: the optical fibers, the transducer's signal wires, and the micromotor's wires.

In the optical fiber-transducer module [Figure 1(b)] is an ultrasonic transducer $\left(\mathrm{LiNbO}_{3}, 40 \mathrm{MHz}\right.$ central frequency) made in-house) that has a hole along its axis. This transducer detects photoacoustic signals. The transducer's piezoelement area has a $2.0-\mathrm{mm}$ outer diameter and its hole has a $0.5-\mathrm{mm}$ diameter. The photoacoustic signal from the transducer is sent to a low-noise amplifier via a micro coaxial cable ( $50 \Omega, 0.44 \mathrm{~mm}$ thick, Hitachi Cable Manchester). A multimode optical fiber with a core diameter of $365 \mu \mathrm{m}(0.22 \mathrm{NA})$ delivers light and is placed along the transducer's central hole. The illumination optical fiber has no light focusing optics, such a lens, so the emitted laser beam diverges with the half angle of 9.6 degrees at the water medium and forms a spot diameter of $1.55 \mathrm{~mm}$ when it passes through the plastic imaging membrane.

In the scanning mirror module [Figure 1(c)], a dielectric-coated fused silica mirror with an outer diameter of $3.0 \mathrm{~mm}$ was connected with a magnet through a metal shaft. The mirror's surface and substrate material provide an excellent condition for both light and ultrasonic wave reflection. Its surface normal shows a 45-degree deflection to the endoscopic probe's axis. Because water and glass have a high sound-propagation speed ratio (1.5/5.1, longitudinal wave; $1.5 / 3.3$, shear wave), the scanning mirror exhibits total internal acoustic reflection within the acceptance angles of the ultrasonic transducer.

The geared micromotor module [Figure 1(d)] is composed of a DC micromotor with a gearbox (gear ratio, 254:1; Namiki Precision, Inc.). Its outer diameter is $1.5 \mathrm{~mm}$ and it is $12.0 \mathrm{~mm}$ long. To provide magnetic coupling with the magnet described in the scanning mirror module [Figure 1(c)], a similar magnet was attached at the tip of the motor's shaft.

To create the probe's housing material, we utilized a stainless steel tube as shown in Figure 1(e). The optical fibertransducer, the scanning mirror, and the geared micromotor modules are placed into the tube as shown in Figure 1(b) through (d). As mentioned, a side portion of the stainless steel tube (corresponding to about a 250-degree angle) was optically and acoustically opened and sealed with the plastic (LDPE) imaging membrane. However, the other unopened rigid stainless wall (corresponding to about 110-degree angle) acts as a connector, or bridge, between the transducer module and the scanning mirror-micromotor modules. Although the proposed system configuration [Figure 1(a)] enables full angular scanning, the real imaging zone in the implemented probe [Figure 1(e)] is partially blocked and the angular field of view is about 250 degrees. The micromotor's electric wires run along the blocked stainless steel bridge. Radial imaging depth is mainly determined by the penetration depth of employed laser beams and the attenuation of acoustic waves. In this study, we could achieve a radial imaging depth of more than $3.0 \mathrm{~mm}$.

\subsection{Peripheral systems and C-scan (pullback) mechanism}

Figure 2 presents a schematic of the photoacoustic endoscopic probe and its peripheral systems. As mentioned, the built-in geared micromotor is a mechanical source and enables radial B-scans. Here the micromotor is controlled by a 
driver circuit, with the driver generating one TTL clock per one micromotor rotation, which corresponds to one angular step (A-line) of the scanning mirror. Since the geared part has a 254:1 gear ratio, the driver generates 254 TTL clocks per one full scanning mirror rotation (B-scan) and A-line step size becomes about 1.42 degrees $\left(=360^{\circ} / 254\right)$. We used these TTL clocks to trigger both the laser system (Nd:YLF) and data acquisition system. To perform C-scans, we used a counter to keep track of the number of TTL clocks generated from the motor driver. After the endoscope performs Bscans at a certain position, the counter sends a pullback signal to the linear stage. By repeating B-scans with the translation motion of the probe as shown in Figure 2, we could achieve C-scans that allow volumetric image production.

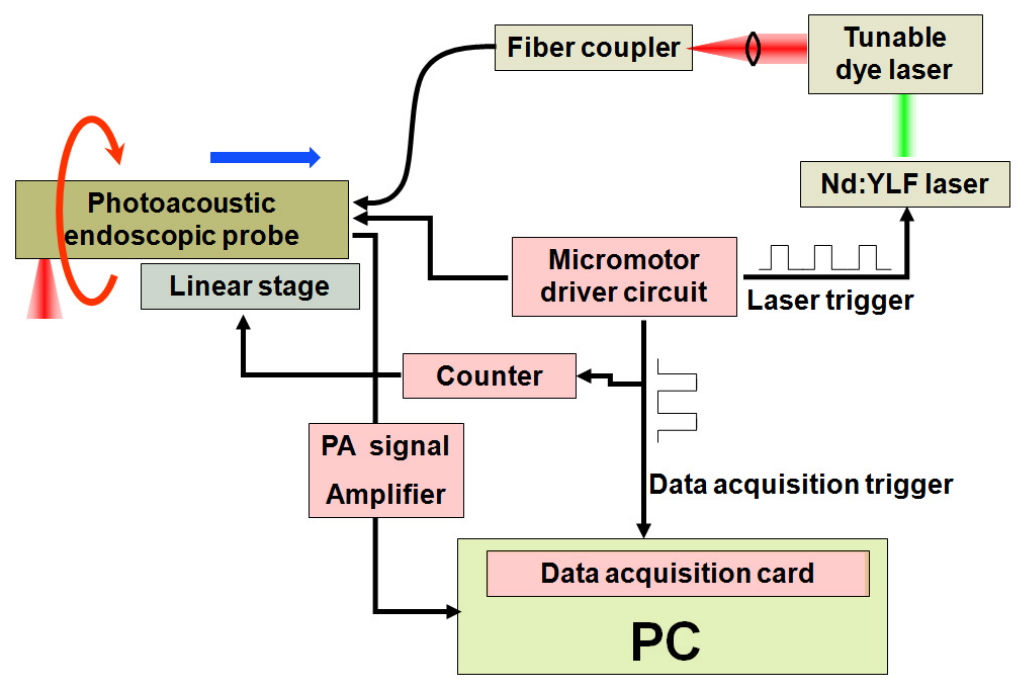

Figure 2: Schematic of the photoacoustic endoscopic probe and its peripheral systems.

A tunable dye laser system pumped by a Nd:YLF laser can allow laser beams with a specific wavelength to be produced and guided to the endoscopic probe through the multimode optical fiber. Detected photoacoustic signals are acquired by a high speed data acquisition card at a sampling rate of $200 \mathrm{MHz}$ ( $5 \mathrm{~ns}$ per data point) after signal amplification. For the signal amplification, we used two serial low-noise amplifiers with the total gains of $48 \mathrm{~dB}$ (ZFL-500LN+, MiniCircuits).

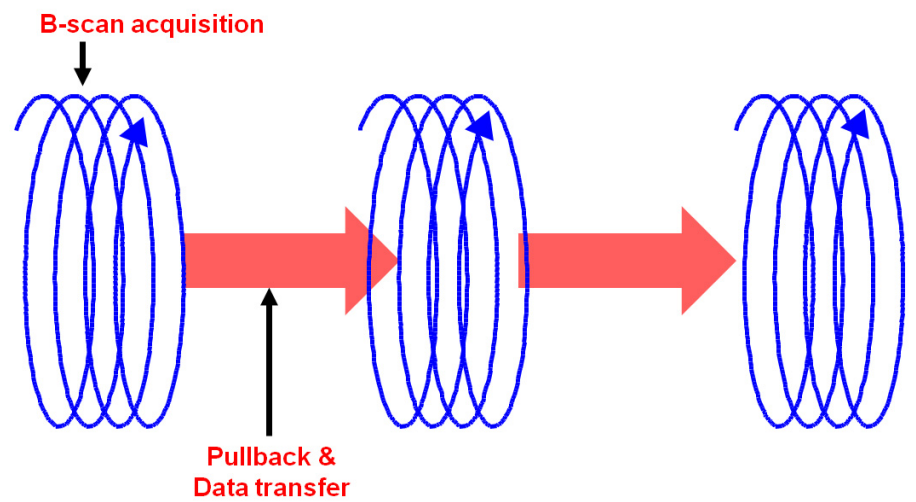

Figure 3: A schematic showing a sequence of B-scan, pullback, and data transfer.

In the experiment, the laser beams' power did not exceed the ANSI safety $\operatorname{limit}^{24}\left(20 \mathrm{~mJ} / \mathrm{cm}^{2}\right.$ per pulse) at the sample's surface (i.e., the plastic membrane). The scanning mirror's rotation speed was set at about 3 frames per second. In the data acquisition, we employed a hardware-based band pass filter $(1-100 \mathrm{MHz})$ and then applied a digital band pass filter $(10-70 \mathrm{MHz})$ to the acquired A-lines. 


\subsection{Metal wire phantom and animal experiment}

To test the system's C-scan ability, we created a metal structure (shown in Figure 4) made of ordinary 0.5-1.0-mm thick metal wires to mimic a stent which is one of common devices in coronary intervention ${ }^{25-26}$. The mesh of the metal structure was formed at about a 6-mm radial distance from the axis. The structure was fixed stably during C-scan experiments and scanned by the endoscopic probe both internally and along the axis. We provided acoustic matching (propagation) medium by spreading enough ultrasound gel over the entire phantom and probe. In this experiment, 100 B-scan slices were acquired with the pullback step size of $160 \mu \mathrm{m}$.

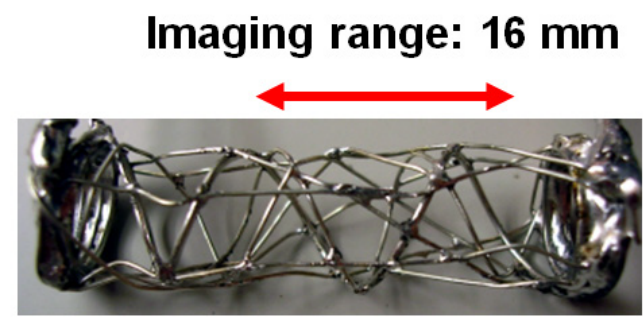

Figure 4: Photo showing the metal wire phantom. The mesh was made of 0.5-1.0 mm thick metal wires.

To demonstrate the system's C-scan capability for actual biological tissues, we imaged a rat rectum in situ as shown in Figure 5. First we sacrificed a rat (Sprague Dawley rats; $\sim 50 \mathrm{~g}$; Harlan National Customer Service Center) by administering a chemical over dose (Pentobarbital, $120 \mathrm{mg} / \mathrm{kg}$, IP). Before introducing the probe, we then spread ultrasound gel to the endoscopic probe and the rectal tract to provide an acoustic matching condition between the probe and rectal tract.

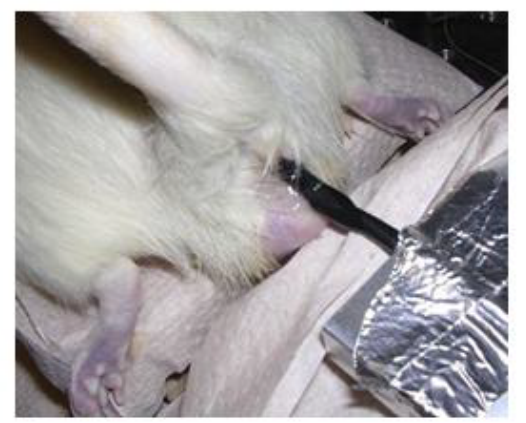

Figure 5: Photo showing in situ rat rectum imaging. The endoscopic probe was inserted into the intact rectal tract and its proximal part was fixed on the linear stage for pullback (C-scan).

All experimental animal procedures were carried out in compliance with the guidelines of the U.S. National Institute of Health. The laboratory animal protocol for this research was approved by the Animal Study Committee of Washington University in St. Louis.

\section{DATA AND RESULTS}

\subsection{Metal wire phantom images}

The endoscopic probe's image resolutions were experimentally measured and reported in a recent paper ${ }^{16}$. Briefly, the radial imaging resolution with the unfocused transducer showed an almost constant value of $\sim 50 \mu \mathrm{m}$ in both clear and turbid ( $0.25 \%$ intralipid) media over a $3.0-\mathrm{mm}$ radial distance (measured from the endoscopic probe's plastic membrane surface). However, the transverse resolution showed target distance dependence; it ranged from about $230 \mu \mathrm{m}$ to 450 $\mu \mathrm{m}$ for the clear medium, and from $177 \mu \mathrm{m}$ to $520 \mu \mathrm{m}$ for the turbid medium. 
Figure 6 shows representative photoacoustic B-scan images of the metal wire phantom acquired at 570-nm wavelength. The left image (a) is a Cartesian coordinate representation of raw data (bipolar signal), the middle image (b) is a Hilberttransformed image of (a), and the right image (c) is a polar coordinate representation of (b). About 78 A-lines, which correspond to the blocked zone (110-degree region) via the stainless steel wall (the bridge), are not included in this image because signals from the metal wall are very strong compared to those from the imaging zone (250-degree region).

(a)

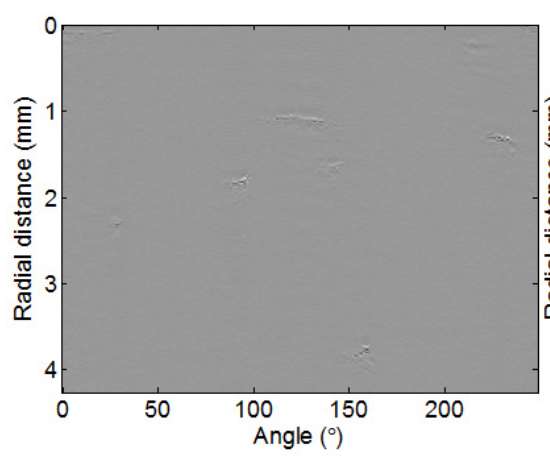

(b)

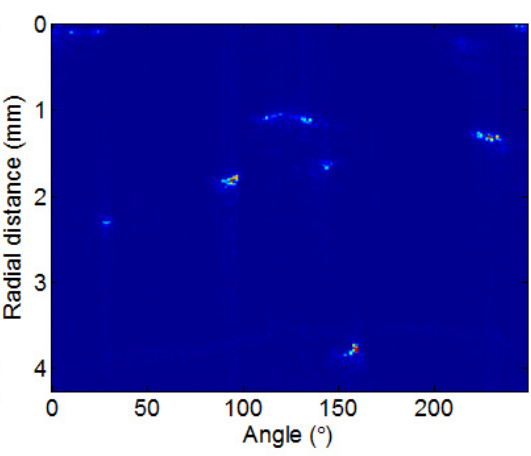

(c)

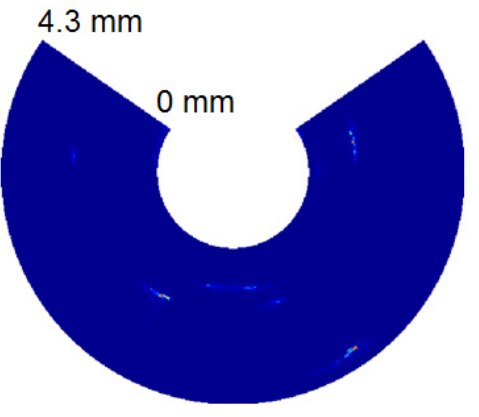

Figure 6: (a) Representative B-scan image, acquired from the metal wire phantom, plotted in Cartesian coordinates. (b) Hilberttransformed image of (a). (c) Polar coordinates representation of image (b).

The acquired 100 B-scan slices (with a step size of $160 \mu \mathrm{m}$ ) were three-dimensionally reconstructed. Three representative images captured at different angles are shown in Figure 7. In Figure 7(a), strong signals from the stainless steel bridge's edge parts are marked with red arrows and one can also see the plastic membrane's acoustically reverberated image.

(a)

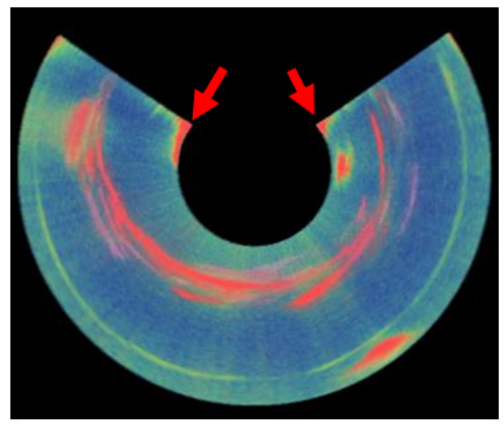

(b)

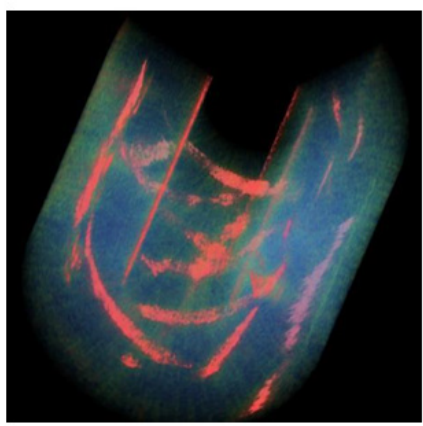

(c)

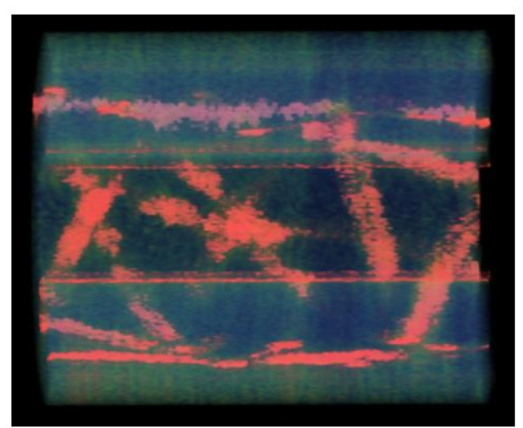

Figure 7: Three-dimensionally rendered images of the wire phantom at three different viewing angles, (a) $\sim(\mathrm{c})$. The endoscopic probe's surrounding space and the metal wire phantom were filled with ultrasound gel. One hundred B-scan slices with $160 \mu \mathrm{m}$ step size were reconstructed.

\subsection{In situ imaging results of rat rectum}

Like the metal wire phantom imaging experiment, we acquired $100 \mathrm{~B}$-scan slices from the rat rectum with a pullback step size of $160 \mu \mathrm{m}$. A representative B-scan image plotted in Cartesian coordinates is shown in Figure 8(a), and its Hilbert-transformed image and polar coordinates representation are shown in Figures 8(b) and (c), respectively. The images show strong photoacoustic signals from blood vessels. From these images, we could estimate the rectum's wall thickness at about $1.0 \mathrm{~mm}$. 
(a)

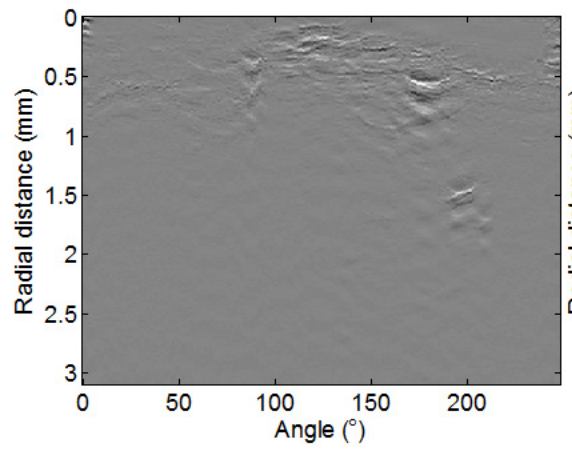

(b)

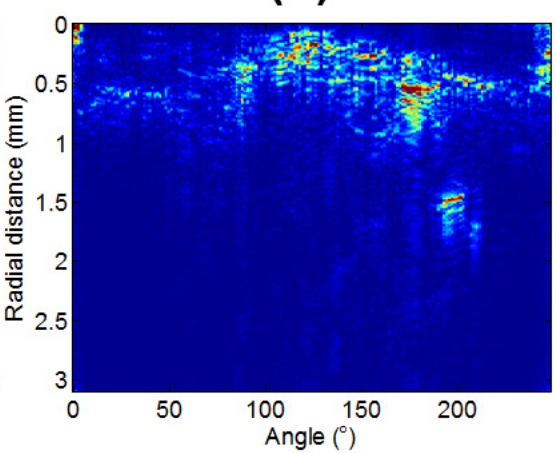

(c)

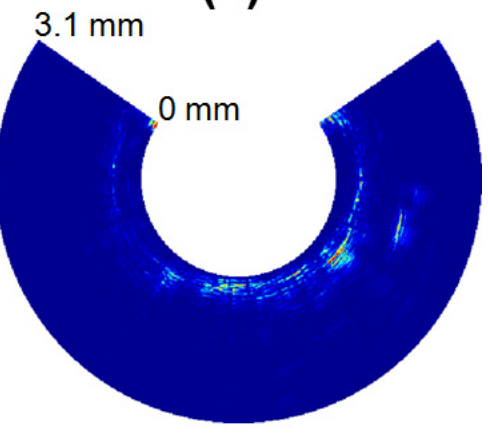

Figure 8: (a) Representative B-scan image, acquired from the rat rectum, plotted in Cartesian coordinates. (b) Hilbert-transformed image of (a). (c) Polar coordinates representation of image (b).

In Figure 9, we present a three-dimensionally rendered rectum image composed with the 100 B-scan slices. To see vascular structure more clearly, we adjusted the transparency of the images. This image can reveal a better understanding of the three-dimensional vascular structure of the intact rectum tract.

(a)

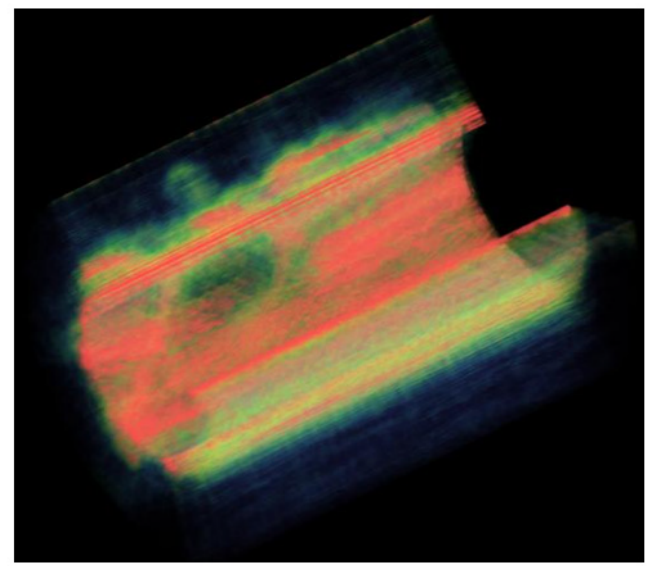

(b)

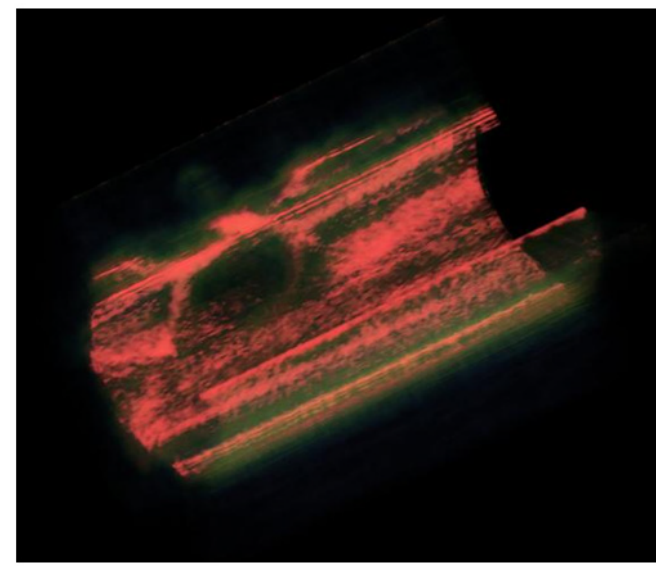

Figure 9: Three-dimensionally rendered images of the wire phantom at two different transparencies, (a): low and (b): high.

\section{SUMMARY AND DISCUSSION}

This paper presents a scanning mirror-based, side-scanning photoacoustic endoscopic probe and its peripheral systems, developed to produce volumetric images of internal organs via C-scans. The in situ rat rectum imaging experiment has demonstrated the probe's actual biological tissue imaging ability. With the serially acquired B-scan slices, we could successfully produce three-dimensional structural images of the vasculature in a rat rectum. Here, the probe's transverse resolution is mainly limited by the employed endoscopic probe's unfocused transducer, but it can be further improved by applying an acoustic lens to the transducer. The current system could allow multi-wavelength imaging through the selection of appropriate laser wavelengths. However, it is necessary to co-register two wavelength data sets at the same position to produce more reliable spectroscopic images that include functional information. In the experiment, the Bscan frame rate was set at $\sim 3 \mathrm{~Hz}$, however it was mainly limited by the micromotor's rotation speed, the laser system's pulse repetition rate, and related data acquisition issues. This study shows the technique's potential for real-time visualization of the structure and function of internal organs. Since conventional pulse-echo based ultrasound endoscopy is sensitive only to the mechanical properties of tissue, photoacoustic endoscopy with spectral volumetric 
imaging is expected to be used as a new diagnostic tool to better characterize tissue abnormalities at super-depths (depths beyond one transport mean free path).

\section{ACKNOWLEDGEMENT}

This research was supported by the National Institutes of Health grants R01 NS46214 (BRP), R01 EB000712, R01 EB008085, and U54 CA136398 (Network for Translational Research). L.W. has a financial interest in Endra, Inc., which, however, did not support this work. J.-M. Y. was supported in part by a Korea Research Foundation (KRF) grant funded by the Korean Government (KRF-2007-357-C00039).The authors are grateful to Namiki Precision, Inc. for supporting the micromotors and Hitachi Cable Manchester for supporting the micro-coaxial cable.

\section{REFERENCES}

[1] C. Strimike, "CE Credit: New Procedures: Understanding Intravascular Ultrasound,” Am. J. Nurs. 96 (6), pp. 40-44 (1996).

[2] S. J. Nicholls, I. Sipahi, P. Schoenhagen, T. Crowe, E. M. Tuzcu, and S. E. Nissen, "Application of intravascular ultrasound in anti-atherosclerotic drug development," Nat. Rev. Drug Discov. 5, pp. 485-492 (2006).

[3] A. Konig, M P. Margolis, R. Virmani, D. Holmes, and V. Klauss, "Technology Insight: in vivo coronary plaque classification by intravascular ultrasonography radiofrequency analysis," Nat. Clin. Pract. Card. 5, pp. 219-229 (2008).

[4] G. Sarno, A. Lerman, J.-H. Bae, C. Schukro, D. Glogar, P. M Margolis, M. Goethals, S. Verstreken, J. Bartunek, A. Koenig, W. Wijns, and M. Vanderheyden, "Multicenter assessment of coronary allograft vasculopathy by intravascular ultrasound-derived analysis of plaque composition," Nat. Clin. Pract. Card. 6, pp. 61-69 (2009).

[5] J. Menzel, W. Domschke, "Gastrointestinal Miniprobe Sonography: The Current Status," Am. J. Gastroeneterol. 95, pp. 605-616 (2000).

[6] A. Fritscher-Ravens, M. Mylonaki, A. Pantes, T. Topalidis, F. Thonke, and P. Swain, "Endoscopic UltrasoundGuided Biopsy for the Diagnosis of Focal Lesions of the Spleen,” Am. J. Gastroeneterol. 97, pp. 2768-2775 (2002).

[7] R. Kiesslich, J. Burg, M. Vieth, J. Gnaendiger, M. Enders, P. Delaney, A. Polglase, W. McLaren, D. Janell, S. Thomas, B. Nafe, P. R. Galle, and M. F. Neurath, "Confocal laser endoscopy for diagnosing intraepithelial neoplasias and colorectal cancer in vivo," Gastroenterol. 127, pp. 706-713 (2004).

[8] A. L. Polglase, W. J. McLaren, S. A. Skinner, R. Kiesslich. M. F. Neurath, and P. M. Delaney, "A fluorescence confocal endomicroscope for in vivo microscopy of the upper- and the lower-GI tract," Gastrointest. Endosc. 62(5), pp. 686-695 (2005).

[9] G. J. Tearney, S. A. Boppart, B. E. Bouma, M. E. Brezinski, N. J. Weissman, J. F. Southern, and J. G. Fujimoto, "Scanning single-mode fiber optic catheter-endoscope for optical coherence tomography," Opt. Lett. 21, pp. 543-545 (1996).

[10] G. J. Tearney, M. E. Brezinski, B. E. Bouma, S. A. Boppart, C. Pitris, J. F. Southern, and J. G. Fujimoto, "In vivo endoscopic optical biopsy with optical coherence tomography," Science 276, pp. 2037-2039 (1997).

[11] Y. Pan, H. Xie, G. K. Fedder, "Endoscopic optical coherence tomography based on a microelectromechanical mirror," Opt. Lett. 26 (24), pp. 1966-1968 (2001).

[12] W. Jung, D. T. McCormick, Y.-C. Ahn, A. Sepehr, M. Brenner, B. Wong, N. C. Tien, and Z. Chen, "In vivo threedimensional spectral domain endoscopic optical coherence tomography using a microelectromechanical system mirror," Opt. Lett. 32 (22), pp. 3239-3241 (2007).

[13] S. H. Yun, G. J. Tearney, B. J. Vakoc, M. Shishkov, W. Y. Oh, A. Desjardins, R. Chan, M. Suter, J. Evans, I. K. Jang, N. S. Nishioka, J. F. de Boer, and B. E. Bouma, "Comprehensive volumetric optical microscopy in vivo," Nat. Med. 12, pp. 1429-1433 (2006).

[14] L. V. Wang, "Prospects of photoacoustic tomography," Med. Phys. 35 (12), pp. 5758-5767 (2008).

[15] J.-M. Yang, K. Maslov, H.-C. Yang, Q. Zhou, L. V. Wang, "Endoscopic photoacoustic microscopy," Proc. SPIE $7177,71770 \mathrm{~N}(2009)$.

[16] J.-M. Yang, K. Maslov, H.-C. Yang, Q. Zhou, K. K. Shung, L. V. Wang, "Photoacoustic endoscopy," Opt. Lett. 34, pp. 1591-1593 (2009).

[17] L. V. Wang, Photoacoustic Imaging and Spectroscopy: Taylor \& Francis/CRC Press, 2009. 
[18] L. V. Wang, "Multiscale photoacoustic microscopy and computed tomography," Nat. Photonics 3(9), pp. 503-509 (2009).

[19] M. Xu, L. V. Wang, "Photoacoustic imaging in biomedicine," Rev. Sci. Instrum. 77, pp. 041101-(1-22) (2006).

[20] H. F. Zhang, K. Maslov, G. Stoica, and L. V. Wang, "Functional photoacoustic microscopy for high-resolution and noninvasive in vivo imaging," Nat. Biotech. 24, pp. 848-851 (2006).

[21] K. H. Song and L. V. Wang, "Deep reflection-mode photoacoustic imaging of biological tissue," J Biomed Opt. 12, pp. 060503 (2007).

[22] K. H. Song, C. H. Kim, C. M. Cobley, Y. N. Xia, and L. V. Wang, "Near-infrared gold nanocages as a new class of tracers for photoacoustic sentinel lymph node mapping on a rat model," Nano Lett. 9 (1), pp. 183-188 (2009).

[23] M. L. Li, J. T. Oh, X. Y. Xie, G. Ku, W. Wang, C. Li, G. Lungu, G. Stoica, and L. V. Wang, "Simultaneous molecular and hypoxia imaging of brain tumors in vivo using spectroscopic photoacoustic tomography," Proc. of IEEE 96 (3), pp. 481-489 (2008).

[24] American National Standards Institute, "American National Standard for the Safe Use of Lasers," ANSI Standard Z136.1, New York (2000).

[25] D. L. Fischman, M. B. Leon, D. S. Baim, R. A. Schatz, M. P. Savage, I. Penn, K. Detre, L. Veltri, D. Ricci, M. Nobuyoshi, et al., "A randomized comparison of coronary-stent placement and balloon angioplasty in the treatment of coronary artery disease. Stent Restenosis Study Investigators," N. Engl. J Med. 331(8), pp. 496-501 (1994).

[26] J. L. Su, B. Wang, S. Y. Emelianov, "Photoacoustic imaging of coronary artery stents," Opt. Exp. 17(22), pp. 19894-901 (2009). 